\title{
Efectividad analgésica postoperatoria de la adición de morfina más dexametasona al anestésico local en bloqueo ecodirigido de plexo braquial
}

\section{Postoperative analgesic effectiveness of the addition of mor- phine plus dexamethasone to the local anesthetic in ultra- sound-guided brachial plexus block}

Henry Jair Mayorga Anaya', Héctor Julio Meléndez Flórez², Carlos Alberto Ortiz Anaya², Mayra Alejandra Meléndez Gómez ${ }^{4}$

\begin{abstract}
Objective: To describe and evaluate the postoperative analgesic effectiveness of the combination of morphine, dexamethasone and local anesthetic in ultrasound-guided brachial plexus block. Materials and Methods: A prospective, observational and analytical cohort study was conducted. The cohort was composed of 106 patients divided into three groups: 1. Local anesthetic (AL), 2. Local anesthetic plus dexamethasone (ALD) and 3. Local anesthetic plus dexamethasone and morphine (ALDM). The outcome variable was acute postope-
\end{abstract}

\section{Key words:}

Brachial plexus, dexamethasone, morphine, postoperative pain

Médico Anestesiólogo. Hospital Universitario de Santander. Profesor Escuela Medicina Universidad Industrial de Santander (UIS).

2 Médico Anestesiólogo. Especialista en Cuidado Crítico. Magíster en Epidemiología. Profesor Titular Departamento de Cirugía, Universidad Industrial de Santander (UIS).

3 Médico Anestesiólogo. Especialista en Docencia Universitaria. Profesor Asociado Departamento de Cirugía (UIS).

4 Médico Residente Postgrado Anestesiología, Universidad Industrial de Santander (UIS).

Fecha de recepción: 25 de junio de 2018

Fecha de aceptación: 22 de noviembre de 2018

\section{ORCID}

https://orcid.org/0000-0002-3699-812

Fuente de financiamiento: Los autores.

Conflicto de interés: Los autores manifestamos no tener ningún conflicto de interés para la realización y divulgación del presente trabajo.

\section{Correspondencia:}

Henry Jair Mayorga Anaya

Carrera 32c No 17 - 87. Bucaramanga, Colombia.

Celular: +573203292935

Email: hjair_16@hotmail.com 
rative pain (DAP) and the need for analgesic rescue. The DAP was evaluated at 3, 6, 9, 12, 18 and 24 postoperative hours using an analogous verbal scale (VAS) and was additionally recorded if it required analgesic rescue. Additionally, latency time, duration of sensory and motor block were recorded. Analysis was done with stata14 software. Results: The overall incidence of postoperative pain was higher in the AL group (89\%) than in the ALD group (58.3\%) and ALDM $(60 \%)$. At 3,6 and 9 hours postoperatively, no differences were found between the three groups. At 12 and 18 hours postoperatively, the incidence of pain in the ALD and ALDM groups was lower and significant $(p<0.05)$ with respect to the AL group. There were no statistically significant differences between the ALD and ALDM groups. At 24 post-operative time no statistically, significant differences were found between the three groups, however at that time the incidence of pain in the AL group was $38 \%$ vs $25 \%$ of the ALDM group vs $23 \%$ of the ALD group. At the end of the study, the intervention in the ALDM group and the ALD group presented Relative Risks (RR) of 0.68 and 0.65 respectively to the LA group. Conclusions: The addition of morphine and dexamethasone or morphine alone to the local anesthetic reduces the postoperative acute pain between 12 and 18 hours and prolongs the time of peripheral ultrasound-guided brachial plexus block. To evaluate differences between these coadjutant's, a study with more power and controlled clinical trial type is required.

\section{RESUMEN}

Objetivo: Describir y evaluar la efectividad analgésica postoperatoria de la combinación de morfina y dexametasona como coadyuvantes a anestésicos locales en bloqueo ecodirigido del plexo braquial. Materiales y Métodos: Se realizó un estudio tipo cohorte prospectivo, observacional y analítico. La cohorte quedó conformada por 106 pacientes divididos en tres grupos: grupo anestésico local sin coadyuvantes (ALSC), grupo anestésico local más dexametasona (ALD) y grupo anestésico local más dexametasona y morfina (ALDM). La variable resultado fue dolor agudo posoperatorio (DAP) moderado a severo y necesidad de rescate analgésico. EL DAP se evaluó a las 3, 6, 9, 12, 18 y 24 horas posoperatorias (POP) utilizando escala verbal análoga (EVA) y, adicionalmente, se registró si se requirió rescate analgésico. Igualmente, se registró tiempo de latencia, duración del bloqueo sensitivo y motor. El análisis se realizó con software stata14. Resultados: La incidencia global de DAP moderado a severo fue mayor en el grupo ALSC (89\%) con respecto al grupo ALD (58,3\%) y grupo ALDM (60\%). A las 3, 6 y 9 horas posoperatorias no se evidenció diferencias entre los tres grupos. A las 12 y 18 horas posoperatorias la incidencia de dolor en los grupos con coadyuvantes fue menor y significativo $(p<0,05)$ con respecto al grupo control. Entre los grupos ALD y ALDM no hubo diferencias estadísticamente significativas. A las 24 horas POP no se encontraron diferencias estadísticamente significativas entre los tres grupos, sin embargo, en ese momento la incidencia de DAP moderado a severo en los tres grupos fue $38 \%, 25 \%$ y $23 \%$ respectivamente. Al final del estudio la intervención en los grupos ALD y ALDM presentaron riesgos relativos (RR) de 0,68 y 0,65 respecto al grupo ALSC. Conclusiones: La adición de morfina y dexametasona o de morfina sola al anestésico local parece disminuir el DAP moderado a severo entre las 12 y 18 horas y prolongar la duración de bloqueos periféricos ecoguiados del plexo braquial. Para evaluar diferencias entre estos coadyuvantes se requieren un estudio con más poder y de tipo ensayo clínico controlado.

\section{Palabras clave:}

Plexo braquial, dexametasona, morfina, dolor posoperatorio 


\section{Introducción}

I abordaje anestésico de pacientes, quienes van a ser sometidos a procedimientos quirúrgicos de extremidades superiores puede realizarse mediante un bloqueo de plexo braquial por vía supraclavicular, infraclavicular, interescalenico y axilar[1]. La introducción de la ecografía para guiar bloqueos locorregionales trajo consigo nuevas herramientas y perspectivas en la práctica de esta disciplina ya que permite la adecuada identificación de los nervios, las estructuras adyacentes, el control de la distribución del anestésico local en tiempo real y la disminución de la probabilidad de toxicidad por éstos[2]-[5].

Las cirugías de miembro superior son procedimiento que frecuentemente se pueden acompañar de dolor agudo postoperatorio (DAP) moderado a severo, requiriendo para su manejo un enfoque multimodal incluyendo el uso de bloqueos de nervios periféricos con anestésicos locales[1],[6].

Estas técnicas anestésicas y/o analgésicas locorregionales tienen como principio fisiológico el bloqueo periférico de estructuras nerviosas por anestésicos locales, han venido en constante evolución en búsqueda de herramientas eficaces para brindar y prolongar una adecuada analgesia posoperatoria. Aparte de los ALs, otros fármacos han sido utilizados como coadyuvantes junto con estos últimos a nivel perineural. Entre estos agentes incluyen opioides, corticoides, neostigmina y/o dexmedetomidina. No obstante, los resultados son contradictorios en la mayoría de los ca$\operatorname{sos}[7],[8],[9]$.

Desde finales de los 80 se han realizado numerosos estudios evaluando el efecto de la morfina perineural en bloqueos nerviosos periféricos, encontrando analgesia hasta por 21 horas[10]. No obstante, otros estudios no han mostrado diferencias significativas respecto a su administración sistémica posterior a realizar bloqueos axilares[11].

El efecto analgésico de los opioides está determinado por su acción a nivel del sistema nervioso central y su posible interacción con receptores periféricos en la mediación de la antinocicepción opioide[12],[13],[14].

Los esteroides tipo dexametasona aplicados vía perineural han logrado demostrar que prolonga la analgesia posoperatoria con mínimos eventos adversos[7],[15],[16],[17]. El mecanismo de acción de dexametasona a nivel periférico no está claramente definido. Sin embargo, se describen tres posibles teorías[7]:

1. Disminución de la actividad nociceptiva de las Fibras $C$ por un efecto directo sobre receptores glucocorticoides y de canales inhibitorios de potasio.
2. Efecto vasoconstrictor local que resulta en una menor absorción de anestésico local.

3. Efecto antiinflamatorio sistémico posterior a la captación vascular del medicamento.

Con estos antecedentes, nuestro objetivo fue describir y evaluar si la combinación de morfina, dexametasona y anestésico local en bloqueo ecodirigido del plexo braquial es efectiva para el control del DAP en cirugías de extremidad superior.

\section{Materiales y Métodos}

Se realizó un estudio de cohorte, prospectivo y analítico durante los meses de marzo de 2016 y julio de 2017 . Se reclutaron pacientes que iban a ser intervenidos quirúrgicamente en miembros superiores y sometidos a técnica anestésica regional con bloqueo de plexo braquial guiado por ecografía. Una vez cumplían criterios de inclusión (cuáles fueron los criterios de inclusión), no tenía exclusión (cuáles fueron los criterios de exclusión) y a criterio del anestesiólogo a cargo quien definía la técnica anestésica y analgésica de bloqueo ecoguiado de plexo braquial, se aplicaba el instrumento de recolección de datos según el grupo y la técnica descrita. Se hizo seguimiento a las $3,6,9,12,18$ y 24 horas posoperatorias (POP) con visita clínica y evaluación por parte de investigadores quienes determinaban el manejo analgésico POP. Se definió DAP moderado a severo al reporte de un valor $\geq 4$ en la escala verbal análoga (EVA) de 11 puntos desde 0 a 10 donde 0 representa ausencia de dolor y 10 el peor dolor imaginable[21] durante el seguimiento. Los datos se registraron y digitaron en una base de datos Excel por duplicado, se verificaron y finalmente se depuraron hasta obtener una sola base de datos, y se analizó con Stata ${ }^{\circledR} 14$. El trabajo fue aprobado por Comité de Ética en Investigación Científica - CEINCI de la Universidad Industrial de Santander y del Hospital Universitario de Santander. Se consideró una investigación sin riesgo en donde los datos se obtuvieron de la historia clínica, del instrumento de recolección y del registro de anestesia, por lo cual no requirió de consentimiento informado adicional al del acto anestésico y quirúrgico usual como se estipula en la Declaración de Helsinki.

Dosis de las drogas: Por ser un estudio descriptivo-analítico, no se propuso una dosis específica, utilizándose las dosis protocolizadas por el servicio de anestesiología y no se estandarizó la intervención. Se describió y registró las diferentes dosis usadas en cada 
grupo: Dexametasona 4 - 8 mg y morfina 30 a 50 $\mathrm{mcg} / \mathrm{kg}$. Respecto al volumen y mezcla de anestésico local, esta consistió en bupivacaína al 0,5\% (10 cc) más lidocaína al 1 o $2 \%$ para completar un volumen final entre 30 a 35 cc para bloqueo supraclavicular, de 15 - 20 cc para bloqueo Interescalénico y de 5 a 10 cc por cada nervio bloqueado a nivel axilar.

\section{Resultados}

Durante un período de tiempo de 14 meses se realizó la recolección de pacientes y registro de datos. Se analizó un total de 106 pacientes que se distribuyeron en tres grupos: $\mathrm{G} 1$ anestésico local sin coadyuvantes (ALSC $n=37$ ), $G 2$ anestésico local más dexametasona ( $A L D n=36$ ) y el $\mathrm{G} 3$ anestésico local más dexametasona y morfina (ALDM $n=33$ ). El $61,3 \%$
(65 pacientes) fueron de sexo masculino, la edad promedio fue 36,6 años con predominio de pacientes ASA I $(81,1 \%)$. El peso promedio de los pacientes fue de $71,2 \mathrm{~kg}$ (si es posible mejor mencionar el IMC promedio más que el peso). Las demás características basales se describen en la Tabla 1.

En los tres grupos, el abordaje más frecuente fue el supraclavicular, seguido del axilar y el interescalénico, sin diferencias significativas respecto a tipo de bloqueo, volumen anestésico y dosis de bupivacaína entre los grupos, al igual que en la duración del acto quirúrgico. La cirugía más frecuente fue la osteosíntesis (OTS), seguido de tenorrafia y neurorrafia, sin diferencias entre los grupos evaluados (Tabla 2).

El tiempo de latencia para bloqueo sensitivo y motor fue significativamente mayor en el ALDM con respecto a los otros dos grupos $(p<0,0005)$. La duración del bloqueo sensitivo y motor fue mayor y significativa

\begin{tabular}{lccccc}
\multicolumn{7}{c}{ Table 1 } \\
\hline \multirow{2}{*}{ Age } & Groups & Minimum & Maximum & Median & p value \\
& Group 1 & 56 & 87 & 73,60 & 0,096 \\
BMI & Group 2 & 51 & 84 & 70,81 & 0,332 \\
Weight & Group 1 & 23,92 & 36,85 & 29,87 & 0,808 \\
& Group 2 & 25,39 & 41,12 & 30,72 & \\
Tourniquet-induced ischae- & Group 1 & 55 & 99 & 81,40 & 0,556 \\
mia duration (min) & Group 2 & 60 & 110 & 80,73 & 94,98 \\
VAS before blockade & Group 2 & 65 & 125 & 93,15 & 1,000 \\
& Group 1 & 75 & 123 & 5,03 & 5,03 \\
\hline
\end{tabular}

\begin{tabular}{|c|c|c|c|c|c|c|}
\hline \multicolumn{7}{|c|}{ Table 2} \\
\hline & Groups & Minimum & Maximum & Median & $\begin{array}{l}\text { Standard } \\
\text { deviation }\end{array}$ & p value \\
\hline \multirow[t]{2}{*}{ VAS at rest 6 h postop } & Group 1 & 0 & 4 & 2,35 & 1,16 & \multirow[t]{2}{*}{$<0,001$} \\
\hline & Group 2 & 0 & 3 & 1,40 & 0,92 & \\
\hline \multirow[t]{2}{*}{ VAS at rest $12 \mathrm{~h}$ postop } & Group 1 & 1 & 4 & 2,48 & 0,93 & \multirow[t]{2}{*}{0,014} \\
\hline & Group 2 & 1 & 4 & 2,00 & 0,75 & \\
\hline \multirow[t]{2}{*}{ VAS at rest $24 \mathrm{~h}$ postop } & Group 1 & 1 & 4 & 2,00 & 1,06 & \multirow[t]{2}{*}{0,162} \\
\hline & Group 2 & 1 & 4 & 1,68 & 0,99 & \\
\hline \multirow[t]{2}{*}{ VAS in movement $6 \mathrm{~h}$ postoperat } & Group 1 & 1 & 5 & 3,28 & 1,13 & \multirow[t]{2}{*}{$<0,001$} \\
\hline & Group 2 & 1 & 4 & 2,03 & 0,92 & \\
\hline \multirow[t]{2}{*}{ VAS in movement1 $2 \mathrm{~h}$ postoperat } & Group 1 & 2 & 5 & 3,33 & 1,02 & \multirow[t]{2}{*}{0,003} \\
\hline & Group 2 & 2 & 5 & 2,68 & 0,88 & \\
\hline \multirow[t]{2}{*}{ VAS in movement24 h postoperat } & Group 1 & 1 & 4 & 2,45 & 1,01 & \multirow[t]{2}{*}{0,190} \\
\hline & Group 2 & 1 & 4 & 2,13 & 1,18 & \\
\hline
\end{tabular}


para el ALDM con respecto al ALSC con una prolongación de 242 minutos de tiempo de analgesia para éste (Tablas 1 y 3).

La incidencia global de DAP moderado a severo durante el seguimiento fue mayor y significativa en el grupo $A L$ y sin diferencias entre ALD y ALDM. La evaluación periódica, mostró un aumento progresivo en DAP en el grupo ALSC a partir de las 6 horas. Entre las 12 y las 18 horas esta diferencia se hizo significativa. La diferencia absoluta de riesgo (DAR) entre ALSC y ALDM fue de $28,8 \%$ y entre el ALSC y ALD fue de $30,5 \%$. No hubo diferencias estadísticamente significativas en DAP entre los grupos con coadyuvancia, quienes presentaron una DAR de solo 3,6\% (Tabla 3). Al evaluar el riesgo relativo (de que ¿Dolor moderado a severo?) (RR) de los grupos ALD y ALDM respecto al grupo que catalogamos como no expuesto (ALSC). Los grupos expuestos presentaron menores RR en las primeras 9 horas, pero no significativos. Al evaluar el DAP como un todo al final del seguimiento, encontramos que los grupos con coadyuvancia tuvieron RR menores, pero solo fue significativa la evaluación del grupo ALDM versus grupo ALSC $(p<0,005)$ (Tablas 4 y 5). La incidencia de rescate en las primeras 18 horas fue mayor y significativo en el grupo ALSC, relación que se invirtió a las 24 horas. En la evaluación del tipo de analgésico de recate utilizado, el uso de opioide fue significativamente más frecuente en el grupo sin coadyuvantes en todos los períodos evaluados ( $p<$ $0,004)$. El uso de acetaminofeno también predominó en este grupo ( $p<0,001)$, pero sin diferencias significativas respecto a los otros dos. Los eventos adversos documentados fueron: síndrome de Horner en un $28,30 \%$, náuseas $14,1 \%$ y prurito en el $4,7 \%$ de los pacientes sin diferencias significativas entre los gru$\operatorname{pos}(p<0,728)$.

\section{Discusión}

El presente estudio descriptivo de tipo cohorte prospectiva en el cual evaluamos el efecto analgésico de adicionar coadyuvantes a la solución de ALs para bloqueos de plexo braquial, no tiene un nivel de evidencia alto, pero su adecuado diseño metodológico que incluyó el cálculo de muestra (no hubo cálculo de muestra, mejor eliminar esta parte), seguimiento postoperatorio y análisis estadístico nos permiten realizar inferencias que son válidas y aplicables a nuestra población. (lo estadístico es la principal debilidad del trabajo por lo cual es mejor no mencionar mucho esto en la discusión a menos que se describa dentro de las debilidades).

El dolor agudo posoperatorio (DAP) continúa

\begin{tabular}{lccc} 
& Table 3 & & \\
\hline & Group 1 & Group 2 & p value \\
Nausea & $2,5 \%$ & $2,5 \%$ & 1.000 \\
Vomiting & $0 \%$ & $0 \%$ & 0,692 \\
Need for supplemental oxygen via nasal cannula to & $10 \%$ & $7,5 \%$ & \\
get SatO $>$ >95\% & & & \\
\hline
\end{tabular}

\begin{tabular}{cccc} 
Table 4 & \\
\hline & Group 1 & Group 2 & p value \\
Additional analgesia 6 h after TKA & $52,5 \%$ & $10 \%$ & $<0,001$ \\
\hline
\end{tabular}

\begin{tabular}{|c|c|c|c|c|}
\hline \multicolumn{5}{|c|}{ Table 5} \\
\hline & Groups & Median & Standard deviation & $p$ value \\
\hline Total additional analgesia $6 \mathrm{~h}$ after TKA & $\begin{array}{l}\text { Group } 1 \\
\text { Group } 2\end{array}$ & $\begin{array}{l}2,63 \\
0,50\end{array}$ & $\begin{array}{l}2,52 \\
2,50\end{array}$ & $<0,001$ \\
\hline Total additional analgesia $12 \mathrm{~h}$ after TKA & $\begin{array}{l}\text { Group } 1 \\
\text { Group } 2\end{array}$ & $\begin{array}{l}2,13 \\
1,13\end{array}$ & $\begin{array}{l}2,50 \\
2,11\end{array}$ & 0,057 \\
\hline Total additional analgesia $24 \mathrm{~h}$ after TKA & $\begin{array}{l}\text { Group } 1 \\
\text { Group } 2\end{array}$ & $\begin{array}{l}1,78 \\
0,96\end{array}$ & $\begin{array}{l}1,76 \\
1,56\end{array}$ & 0,032 \\
\hline
\end{tabular}


siendo un problema frecuente en las unidades de cuidados postanestésicos. Algunos estudios relevan incidencias que oscilan desde un $11 \%$ hasta un $60 \%$ de la población posquirúrgica[18],[19]. Las cirugías de hombro así como de miembro superior tienen peor control del DAP, encontrándose valores de EVA elevados en el posoperatorio hasta en $66 \%$ de los pacientes[20],[21]. Los datos anteriores son similares a los documentados en nuestro estudio en el cual encontramos incidencias de DAP hasta en $80 \%$ de los pacientes durante el seguimiento postquirúrgico.

Actualmente, se ha descrito el uso de diversos agentes terapéuticos como opioides, neostigmina, clonidina, dexmedetomidina y corticoides como sustancias que pudiesen disminuir la incidencia del dolor posoperatorio y modular su intensidad y actuar como potenciadores del efecto del anestésico local perineural y prolongar su tiempo de acción, sin embargo, los estudios son contradictorios con algunas de estas sustancias[7],[13],[15]. Dados estos reportes nosotros quisimos describir y evaluar en nuestra práctica diaria las distintas opciones terapéuticas de coadyuvantes del anestésico local en bloqueo regional de plexo braquial, utilizando morfina y dexametasona perineural.

El uso de dexametasona perineural tiene resultados favorables como estrategia analgésica. Un reciente metaanálisis que incluyó 2.138 pacientes, concluyó que el uso de $4 \mathrm{mg}$ de dexametasona perineural puede prolongar el tiempo de analgesia posoperatoria entre 6 a 8 horas dependiendo del tipo de anestésico local utilizado[22], resultados similares a los obtenidos en este estudio en el cual hubo una prolongación de 241 minutos con dosis de 4 a 8 mg de dexametasona perineural. En el presente estudio no se encontró interacción entre la dosis de dexametasona y la duración de la analgesia lo cual es consistente con otros estudios realizados[7],[15],[16],[17], sin embargo, el tiempo de latencia para bloqueo completo sensitivo y motor y la calidad del mismo, no fueron afectadas por el uso de esta sustancia.

Adicionalmente documentamos una incidencia global de DAP moderado a severo de $58,3 \%$ en el grupo de dexametasona perineural, lo cual fue menor a la incidencia reportada para el grupo no expuesto (88\%). No documentamos en la literatura valores de incidencia específicos de DAP en este tipo de población quirúrgica sometida a técnicas anestésicas locorregionales ecoguiadas.

De igual forma al seguimiento posoperatorio encontramos puntuaciones menores en la valoración de la intensidad del dolor. Al final del estudio la dexametasona perineural mostró un RR de 0,65 a favor de la intervención, lo cual se correlaciona con lo evidencia- do en los distintos estudios clínicos[7],[22],[23],[24]. Así mismo encontramos que no existen diferencias en cuanto a complicaciones neurológicas y/o eventos adversos con el uso de dexametasona perineural. En cuanto a la analgesia de rescate, observamos un menor consumo de opioides y acetaminofen en el grupo de dexametasona perineural con respecto al grupo control. Estas diferencias se acentúan a la hora 12 y 18 postoperatorias en las cuales se presentan las mayores divergencias en la puntuación de EVA en los grupos evaluados, lo cual sugiere un efecto analgésico de la dexametasona perineural, no obstante los valores de DAP son elevados en ambos grupos descritos, lo que conlleva a plantear la necesidad de propender por un adecuado manejo multimodal del dolor.

El uso de opioides a nivel perineural ha sido objeto de diversas controversias. Murphy y cols[25] en una revisión sistemática encontraron un efecto analgésico con el uso de morfina perineural, similar al reportado por nosotros. Agüero-Martínez y cols, concluyeron que el uso de estos fármacos en conjunto con el AL es eficaz para prolongar el tiempo de analgesia POP y el tiempo de duración del bloqueo en más de 8 horas al ser comparados con el solo uso de AL solo. Igualmente se reporta una mejor evolución del DAP a las 24 horas con morfina perineural[10], resultados que son similares a los nuestros. Al igual que estos estudios descritos, el tiempo de prolongación del bloqueo y analgesia POP fue muy similar en nuestro estudio. A pesar de estos resultados, nosotros no encontramos diferencias estadísticamente significativas entre el grupo ALD y el grupo ALDM en cuanto a la incidencia de dolor, tiempo de duración de bloqueo, analgesia posoperatoria y eventos adversos, lo cual sugiere que uno de los dos coadyudantes podría ser más que suficiente para lograr analgesia. Para evaluar diferencias significativas entre estos dos grupos, se requiere una muestra con mayor poder, dado la mínima diferencia en la incidencia de dolor entre las 12 y 24 horas POP.

Adicionalmente, encontramos mayores tiempos de latencia sensitivo y motor con valores estadísticamente significativos, lo cual es distinto a lo referido en la literatura[10],[27],[28]. Lo anterior podría estar relacionado con los cambios de la constante pka de la mezcla generados por la combinación de estos agentes terapéuticos. No obstante, logramos determinar que este hallazgo no afectó la calidad del bloqueo ni los desenlaces esperados. Al final del estudio la intervención de morfina perineural mostró un RR de 0,68 para aparición del dolor, lo cual favorece su uso como coadyuvante. No hay diferencias en cuanto a la presentación de efectos adversos con lo descrito en la literatura mundial. Finalmente, logramos establecer 
en el grupo de morfina perineural, un menor uso de analgesia de rescate con opioide endovenoso y acetaminofeno oral a la hora 12 y 18 del posoperatorio.

Nuestro estudio no es el gold standard para evaluar eficacia, por eso nos enfocamos a la efectividad. Tampoco estamos libres de sesgos, entre los cuales la falta de uniformidad en las dosis de morfina y dexametasona puede haber influido en los resultados, de no diferencia entre los dos grupos que utilizaron estos medicamentos.

El presente estudio posee varias limitaciones estadísticas y metodológicas que pensamos merecen discusión. Primero que todo, a pesar de que el diseño tiene características muy similares a un ensayo clínico controlado, éste carece de un cálculo de tamaño muestral adecuado, de randomización a grupo de tratamiento y evaluación ciega de los datos. Todo esto representa una limitación mayor en el nivel de evidencia que cualquier estudio puede otorgar. Sin embargo, consideramos que los datos obtenidos facilitarán el diseño estadístico de un estudio randomizado ciego en búsqueda de aclarar el rol individual de coadyuvantes como dexametasona y morfina en nuestra práctica clínica en un futuro próximo. Además, dentro de lo metodológico, reconocemos que el diseño del presente trabajo carece de las características idóneas como modelo de experimentación. En esta línea, en futuros estudios, deberemos estandarizar las técnicas anestésicas, de bloqueo y las dosis de drogas utilizadas para que los grupos sean efectivamente comparables. Adicionalmente, reconocemos que el uso de coadyuvantes perineurales pueden tener un efecto final más bien sistémico y éste es un sesgo que se debe limpiar. Actualmente, la evidencia ya ha determinado que, de manera rotunda que dexametasona perineural es superior a su contraparte endovenosa, sin embargo, el estudio de dexametasona más morfina perineural requerirá al menos un grupo de control de anestésicos locales más una dosis similar de morfina sistémica para dar mayor validez al modelo de ensayo.

\section{Conclusiones}

La adición de morfina y dexametasona al anestésico local en bloqueos ecoguiado de plexo braquial en pacientes sometidos a cirugía de miembro superior parece disminuir el DAP a las 12 y 18 horas poscirugía y no aumentaría el riesgo de eventos adversos en comparación a un bloqueo exclusivo con ALs. No obstante sugerimos la realización de un ensayo clínico controlado para mejorar el nivel de evidencia del uso conjunto de estos coadyuvantes en el manejo del dolor agudo posoperatorio en este tipo de cirugías.

\section{Referencias}

1. Cho $\mathrm{CH}$, Song $\mathrm{KS}$, Min BW, Lee $\mathrm{KJ}$, Ha E, Lee YC, et al.; $\mathrm{CH}$. Multimodal approach to postoperative pain control in patients undergoing rotator cuff repair. Knee Surg Sports Traumatol Arthrosc. 2011 Oct;19(10):17448.

2. Gray AT. Ultrasound-guided regional anesthesia: current state of the art. Anesthesiology. 2006 Feb;104(2):368-73.

3. Sandhu NS, Capan LM. Ultrasound-guided infraclavicular brachial plexus block. Br J Anaesth. 2002 Aug;89(2):254-9.

4. Awad IT, Chan V. Ultrasound imaging of peripheral nerves: a need for a new trend. Reg Anesth Pain Med. 2005 Jul-
Aug:30(4):321-3.

5. Liu SS, Ngeow JE, Yadeau JT. Ultrasound-guided regional anesthesia and analgesia: a qualitative systematic review. Reg Anesth Pain Med. 2009 JanFeb;34(1):47-59.

6. Pinzón O. Anestesia regional para cirugía de miembro superior. Revista Colombiana de Anestesiología 2000; 23 (3):

7. Albrecht E, Kern C, Kirkham KR. A systematic review and meta-analysis of perineural dexamethasone for peripheral nerve blocks. Anaesthesia. 2015 Jan;70(1):71-83.

8. Liu J, Richman KA, Grodofsky SR, Bhatt S, Huffman GR, Kelly JD 4th, et al. Is there a dose response of dexamethasone as adjuvant for supraclavicular brachial plexus nerve block? A prospective randomized doubleblinded clinical study. J Clin Anesth. 2015 May;27(3):237-42.

9. Kirksey MA, Haskins SC, Cheng J, Liu SS. Local Anesthetic Peripheral Nerve Block Adjuvants for Prolongation of Analgesia: A Systematic Qualitative Review. Schwentner $C$, ed. PLOS ONE. 2015;10(9). https:// doi.org/10.1371/journal. pone.0137312.

10. Bazin JE, Massoni C, Bruelle P, Fenies V, Groslier D, Schoeffler P. The addition of opioids to local anaesthetics in brachial plexus block: the comparative effects of morphine, buprenorphine and sufentanil. Anaesthesia. 1997 Sep;52(9):858-62.

11. Bourke DL, Furman WR. Impro- 
ved postoperative analgesia with morphine added to axillary block solution. J Clin Anesth. 1993 Mar-Apr;5(2):114-7.

12. Labuz D, Mousa SA, Schäfer M, Stein C, Machelska H. Relative contribution of peripheral versus central opioid receptors to antinociception. Brain Res. 2007 Jul;1160:30-8.

13. Shannon HE, Lutz EA. Comparison of the peripheral and central effects of the opioid agonists loperamide and morphine in the formalin test in rats. Neuropharmacology. 2002 Feb;42(2):25361.

14. Sabbe MB, Grafe MR, Mjanger E, Tiseo PJ, Hill HF, Yaksh TL. Spinal delivery of sufentanil, alfentanil, and morphine in dogs. Physiologic and toxicologic investigations. Anesthesiology. 1994 Oct;81(4):899-920.

15. Choi S, Rodseth R, McCartney CJ. Effects of dexamethasone as a local anaesthetic adjuvant for brachial plexus block: a systematic review and meta-analysis of randomized trials. $\mathrm{Br} J$ Anaesth. 2014 Mar;112(3):427-39.

16. Biradar PA, Kaimar P, Gopalakrishna K. Effect of dexamethasone added to lidocaine in supraclavicular brachial plexus block: A prospective, randomised, double-blind study. Indian J Anaesth. 2013 Mar;57(2):180-4.

17. Liu J, Richman KA, Grodofsky
SR, Bhatt S, Huffman GR, Kelly JD 4th, et al. Is there a dose response of dexamethasone as adjuvant for supraclavicular brachial plexus nerve block? A prospective randomized doubleblinded clinical study. J Clin Anesth. 2015 May;27(3):237-42.

18. Schug SA, Chong C. Pain management after ambulatory surgery. Curr Opin Anaesthesiol. 2009 Dec;22(6):738-43.

19. Bartusseck E, Fatehi S, Motsch J, Grau T. [Survey on practice of regional anaesthesia in Germany, Austria, and Switzerland. Part 1: quality assurance and training concepts]. Anaesthesist. 2004 Sep;53(9):836-46.

20. Pellegrin C, Bravo D, Lorca M. Prevalencia de dolor posoperatorio de cirugía electiva en el complejo hospitalario San José. Rev. chil. Antes. 2014;43 Suplemento 1:226-34.

21. Morales C, Ortiz R, Martínez A, Echevarría M. Estudio comparativo de la eficacia del bloqueo supraclavicular en la artroscopia de hombro. Rev Soc Esp Dolor. 2010;17(8):366-71.

22. Kirkham $K R$, Jacot-Guillarmod A, Albrecht E. Optimal Dose of Perineural Dexamethasone to Prolong Analgesia After Brachial Plexus Blockade: A Systematic Review and Metaanalysis. Anesth Analg. 2018 Jan;126(1):270-9.
23. Kawanishi $R$, Yamamoto $K$, Tobetto $Y$, Nomura K, Kato M, Go $R$, et al. Perineural but not systemic low-dose dexamethasone prolongs the duration of interscalene block with ropivacaine: a prospective randomized trial. Local Reg Anesth. 2014 Apr;7:5-9.

24. Huynh TM, Marret E, Bonnet F. Combination of dexamethasone and local anaesthetic solution in peripheral nerve blocks: A metaanalysis of randomised controlled trials. Eur J Anaesthesiol. 2015 Nov;32(11):751-8.

25. Murphy DB, McCartney CJ, Chan VW. Novel analgesic adjuncts for brachial plexus block: a systematic review. Anesth Analg. 2000 May;90(5):1122-8.

26. Agüero $M$, Aguado $O$, Vargas R. Bloqueo del plexo braquial. Efecto de la asociación anestésicos locales/opiodes. Ensayo Clínico. Revista de la Sociedad Cubana de anestesiología y reanimación. 2005;4(1): 115-22.

27. Mays KS, Lipman JJ, Schnapp M. Local analgesia without anesthesia using peripheral perineural morphine injections. Anesth Analg. 1987 May;66(5):417-20.

28. Murphy DB, McCartney CJ, Chan VW. Novel analgesic adjuncts for brachial plexus block: a systematic review. Anesth Analg. 2000 May;90(5):1122-8. 